\title{
Comparison of the Efficacy of Different Arterial Waveform- derived Variables (Pulse Pressure Variation, Stroke Volume Variation, Systolic Pressure Variation) for Fluid Responsiveness in Hemodynamically Unstable Mechanically Ventilated Critically Ill Patients
}

\author{
Nitish Kumar ${ }^{1}$, Deepak Malviya ${ }^{2}$, Soumya S Nath ${ }^{3}$, Shivani Rastogi ${ }^{4}$, Vishal Upadhyay ${ }^{5}$
}

\begin{abstract}
Introduction: This study was conducted to assess fluid responsiveness in critically ill patients to avoid various complications of fluid overload. Material and methods: This study was done in an ICU of a tertiary care hospital after approval from the institute ethical committee over 18 months. A total of 54 consenting adult patients were included in the study. Patients were hemodynamically unstable requiring mechanical ventilation, had acute circulatory failure, or those with at least one clinical sign of inadequate tissue perfusion. All patients were ventilated using tidal volume of $6-8 \mathrm{~mL} / \mathrm{kg}, \mathrm{RR}-12-15 /$ minutes, positive end expiratory pressure (PEEP) $-5 \mathrm{~cm}$ of water, and plateau pressure was kept below $30 \mathrm{~cm}$ water. They were sedated throughout the study. The arterial line and the central venous catheter were placed and connected to Vigileo-FloTrac transducer (Edward Lifesciences). Patients were classified into responder and nonresponder groups on the basis of the cardiac index $(\mathrm{Cl})$ after fluid challenge of $10 \mathrm{~mL} / \mathrm{kg}$ of normal saline over 30 minutes. Pulse pressure variation (PPV), stroke volume variation (SVV), and systolic pressure variation (SPV) were assessed and compared at baseline, 30 minutes, and 60 minutes.

Results: In our study we found that PPV and SVV were significantly lower among responders than nonresponders at 30 minutes and insignificant at 60 minutes. Stroke volume variation was $10.28 \pm 1.76$ in the responder compared to $12.28 \pm 4.42(p=0.02)$ at 30 minutes and PPV was $15.28 \pm$ 6.94 in responders while it was $20.03 \pm 4.35$ in nonresponders $(p=0.01)$. We found SPV was insignificant at all time periods among both groups. Conclusion: We can conclude that initial assessment for fluid responsiveness in critically ill mechanically ventilated patients should be based on PPV and SVV to prevent complications of fluid overload and their consequences.

Keywords: Cardiac index, Positive end expiratory pressure, Pulse pressure variation, Stroke volume variation, Systolic pressure variation.

Indian Journal of Critical Care Medicine (2021): 10.5005/jp-journals-10071-23440
\end{abstract}

\section{INTRODUCTION}

Hemodynamic instability is quite common in critically ill patients. ${ }^{1}$ This hemodynamic instability could be due to various reasons like volume insufficiency, septic myocardiopathy, etc. This circulatory insufficiency leads to low cardiac output (CO), which fails to sustain tissue perfusion and oxygenation ${ }^{2}$ leading to anaerobic metabolism, lactic acidosis, organ dysfunction, organ failure, and finally leading to rapid deterioration and death. Administering fluid to these patients may seem to be an easy option, but several studies had demonstrated that only about half of the critically ill hemodynamically unstable patients benefit from fluid loading. ${ }^{3}$ This follows that the other half of the patients might potentially or actually be harmed by any fluid administration. ${ }^{4-6} \mathrm{~A}$ patient may benefit from fluid administration only if there is some preload reserve left. But, often these patients have already been resuscitated partially, so the presence of preload reserve is not guaranteed and further fluid infusion, among other deleterious effects, may promote pulmonary edema, particularly in cases of increased pulmonary permeability. Also, positive cumulative fluid balance had been shown to be an independent risk of death. ${ }^{7}$

It is true that in patients with a high suspicion of severe sepsis or septic shock, several studies emphasized the importance of volume resuscitation in the first hours of management. ${ }^{8,9}$ But, in tertiary
${ }^{1-5}$ Department of Anesthesiology and Critical Care Medicine, Dr Ram Manohar Lohia Institute of Medical Sciences, Lucknow, Uttar Pradesh, India

Corresponding Author:Soumya S Nath, Department of Anesthesiology and Critical Care Medicine, Dr Ram Manohar Lohia Institute of Medical Sciences, Lucknow, Uttar Pradesh, India, Phone: +91 9648935430, e-mail: soumyanath@rediffmail.com

How to cite this article: Kumar N, Malviya D, Nath SS, Rastogi S, Upadhyay V. Comparison of the Efficacy of Different Arterial Waveform-derived Variables (Pulse Pressure Variation, Stroke Volume Variation, Systolic Pressure Variation) for Fluid Responsiveness in Hemodynamically Unstable Mechanically Ventilated Critically III Patients. Indian J Crit Care Med 2021;25(1):48-53.

Source of support: Nil

Conflict of interest: None

care centers, patients are often referred from smaller centers where they have been already somewhat resuscitated. Further volume administration, in sustained hypotension, represents a therapeutic dilemma. In patients with acute lung injury, a restrictive fluid strategy was demonstrated to be better than a liberal fluid strategy 
in terms of ventilator-free and ICU-free days. ${ }^{10}$ Thus, decision to load a patient with fluid requires an accurate assessment of patients' intravascular volume status.

Previously, static hemodynamic parameters like central venous pressure (CVP), pulmonary capillary wedge pressure (PCWP), and right ventricular end-diastolic volume (RVEDV) were used to assess fluid status. Several studies have highlighted that they suffer several fallacies and are insensitive markers of volume status and could be misleading. As a result of these shortcomings, the dynamic parameters of fluid responsiveness have been proposed, which use heart-lung interactions, i.e., respiration-induced changes in preload and afterload, to predict fluid responsiveness. These parameters include stroke volume variation (SVV), pulse pressure variation (PPV), and systolic pressure variation (SPV). ${ }^{11,12}$

In spite of numerous studies, accurate prediction of fluid responsiveness remains one of the most difficult tasks at the bedside and to assess whether the volume expansion will increase patients' CO or not so that hypovolemia as well as hypervolemia can be avoided. So, we planned to do this study to assess and compare the efficacy of different arterial waveformderived variables (PPV, SVV, SPV) for fluid responsiveness in hemodynamically unstable mechanically ventilated critically ill patients in Indian scenario.

\section{Materials and Methods}

This single-centered, interventional, comparative, prospective study was performed after getting approval from the Institutional Ethics Committee (IEC No.: 14/16). Patients who fulfilled the inclusion criteria were enrolled after obtaining an informed consent from the patient's legal guardian. The patient was able to withdraw from the study at any time, without giving any reason and without impact on treatment. The study was conducted in hemodynamically unstable critically ill nonsurgical patients, admitted in ICU. A total of 54 patients were included in the study. The data collection for the study spanned over a period of $1 \frac{1}{2}$ years starting from February 2017 to August 2018.

\section{Inclusion Criteria}

- Hemodynamically unstable patients requiring mechanical ventilation.

- Age $>18$ years of age

- Acute circulatory failure patient for which the decision was taken to administer fluids.

- Presence of at least one clinical sign of inadequate tissue perfusion defined as:

- Systolic blood pressure (SBP) $<90 \mathrm{~mm} \mathrm{Hg}$ or mean arterial pressure (MAP) $<65 \mathrm{~mm} \mathrm{Hg}$

- The need of vasopressor drugs

- Urine output $<0.5 \mathrm{~mL} / \mathrm{kg} /$ hour for atleast 2 hours

- Tachycardia (heart rate $>100 /$ minute)

- Serum lactate ( $>2 \mathrm{mmol} / \mathrm{L})$

- $\mathrm{ScVO}_{2}$ (central venous oxygen saturation) $<70 \%$

\section{Exclusion Criteria}

Patients with irregular cardiac rhythm: Patient with atrial fibrillation (AF) or having frequent ectopics

- Patient with acute respiratory distress syndrome (ARDS)

- Valvular heart disease: Significant aortic or mitral valve lesions

- Spontaneously breathing patients

- Renal patient with oliguria and volume overload.
The sample size was calculated based on previous study ${ }^{13}$ using the following formula: ${ }^{14}$

$$
n=Z_{1-\alpha / 2}^{2} \times \mathrm{SD}^{2} / d^{2}
$$

$Z_{1-\alpha / 2}=$ Power of the study

SD: Assumed standard deviation

$d$ : Absolute error (difference in means)

Assuming $80 \%$ power, 5\% significance level with $95 \%$ confidence interval, and assumed standard deviation being 2 , the total sample size calculated was 41 . We decided to include 50 patients.

\section{Vigileo-FloTrac}

Vigileo-FloTrac (Edwards Life Science, Irvine, CA, United States) allows for automated and continuous monitoring of $\mathrm{CO}$ based on pulse contour analysis and also monitoring of the respiratory variations in stroke volume (SVV). The Vigileo-FloTrac device analyzes the arterial waveform to determine stroke volume (SV). The FloTrac system (Edwards) is a specific pressure transducer attached to an arterial line catheter and connected to a specific monitor (Vigileo). The arterial waveform is assessed at $100 \mathrm{~Hz}$. The standard deviation (SD) of the pulse pressure (PP) is determined over a 20-second period. To calculate $\mathrm{CO}$, the software uses an algorithm based on the relationship between arterial PP and SV and considers vessel compliance and peripheral resistance. Vessel compliance is estimated from nomograms based on age, gender, height, and weight, and peripheral resistance is determined from arterial waveform characteristics. Vigileo-FloTrac devices allow for the determination of the SVV. This index is displayed continuously on the monitor.

Pulse pressure is defined as the difference between systolic and diastolic arterial blood pressure. Maximal $\left(\mathrm{PP}_{\max }\right)$ and minimal $\left(\mathrm{PP}_{\min }\right)$ were determined over the same respiratory cycle.

$$
P P V=\left(P P_{\text {max }}-P P_{\text {min }}\right) /\left[\left(P_{\text {max }}+P_{\text {min }}\right) / 2\right] \times 100
$$

Stroke volume variation-It is the percent of change in SV during inspiration and expiration during the most recent 20 seconds. Stroke volume variation is also defined as the variation of beat-to-beat SV from the mean value during the most recent 20 seconds. It was calculated using the following formula:

$$
\mathrm{SVV}=\left(\mathrm{SV}_{\max }-\mathrm{SV}_{\text {min }}\right) / S \mathrm{SV}_{\text {mean }} \times 100
$$

The mean value of three consecutive SVV determinations will be used for statistical analysis ( $>1$ minute).

Systolic pressure variation is defined as the difference between the maximal and minimal values of systolic arterial pressure recorded over a respiratory cycle.

$$
\mathrm{SPV}=\Delta \mathrm{Up}+\Delta \text { Down normal value }
$$

Sometimes calculated as a fraction by the equation:

$$
\begin{aligned}
\mathrm{SPV} & =\mathrm{SBP}_{\text {max }}-\mathrm{SBP}_{\min } /\left(\mathrm{SBP}_{\max }+\mathrm{SBP}_{\min } / 2\right) \\
\Delta \mathrm{Up} & =\mathrm{SBP}_{\text {max }}-\text { Apneic baseline normal value } \\
\Delta \mathrm{Down} & =\text { Apneic baseline }-\mathrm{SBP}_{\min } \text { normal value }
\end{aligned}
$$

All the selected patients were mechanically ventilated using the volume control mode with tidal volume $6-8 \mathrm{~mL} / \mathrm{kg}$, respiratory 
rate $12-15 /$ minute, PEEP up to $5 \mathrm{~cm} \mathrm{H}_{2} \mathrm{O}$, and plateau pressure

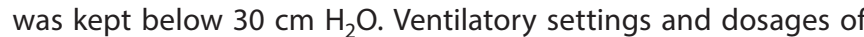
ionotropic and vasopressor drugs were kept constant during the entire study period. The patients remained sedated during the study period using propofol (1-4 mg/kg iv $\mathrm{f} / \mathrm{b}$ infusion of $1-2 \mathrm{mg} /$ $\mathrm{kg} / \mathrm{hour}$ ) so that their spontaneous effort will be masked. An 22G cannula was placed in the radial artery and 7Fr three-lumen CVC was placed in the right internal jugular vein using the Seldinger's technique after taking all aseptic precautions. Both radial artery and CVC were connected to a transducer and later on a dedicated FloTrac transducer was connected to these lines to one end and to the Vigileo system on the other ends. The system enables the continuous monitoring of $\mathrm{CO}$, cardiac index $(\mathrm{CI}), \mathrm{SV}$, and SVV by the pulse contour analysis. The PPV and SPV were calculated using a standard Multipara monitor. These monitors have the features of measuring PPV and SPV in response to fluid replacement therapy. This feature can be used with the standard arterial pressure contour analysis. During the study period, we froze a pressure waveform and identify the maximum and minimum PP and also maximum and minimum systolic pressures, which coincided with the respiration cycles and estimated PPV and SPV by using the standard formula. Both PPV and arterial SPVs values were considered as the average of three consecutive values at a 1-minute interval. Hemodynamic measurements were recorded in the supine position with the all transducers positioned at the level of fourth intercostal space in the mid-axillary line. Three sets of measurements were recorded: The first set was at the baseline ( 0 minute), second after fluid challenge, i.e., 30 minutes, and third at 60 minutes. All selected patients were given $10 \mathrm{~mL} / \mathrm{kg}$ body weight of normal saline as a fluid challenge over 30 minutes. The following hemodynamic variables were recorded: heart rate (HR), CVP, mean arterial blood pressure (MAP), and $\mathrm{Cl}$, and with the help of the multiparametric monitor and using the Vigileo system SVV, PPV, and SPV were measured. Variables obtained with the Vigileo-FloTrac device were sampled every 20 seconds. The values were recorded at baseline ( 0 minute), after fluid challenge at 30 minutes and at 60 minutes, and this was used to classify patients as responders and nonresponders. Following the crystalloid bolus, patients with a $\mathrm{Cl}$ increase of more than $10 \%$ were classified as responders and those with an increase of less than $10 \%$ were classified as nonresponders. ${ }^{15}$ The CVP, HR, MAP, CI, PPV, SVV, and SPV were simultaneously recorded at each time point. The primary objective was to measure PPV, SVV, and SPV before and after fluid challenge to the mechanically ventilated critically ill patients to classify patients as responders and nonresponders based on the percentage change in $\mathrm{Cl}$, and the secondary objectives were to compare and validate the accuracy and predictability of fluid responsiveness measured using PPV, SVV, and SPV.

The statistical analysis was carried out using IBM Corp. (2013) IBM SPSS Statistics for Windows, Version 22.0 (IBM Corp., Armonk, NY).
Data were presented as mean \pm SD. Categorized data were presented as frequency and/or percentage. The parametric data were compared among groups using the one-way analysis of variance and the unpaired $t$-test. Nonparametric data were compared using the Pearson's chi-square test.

\section{Results}

A total of 54 patients were recruited. Of these, two patients had technical issue as their arterial line got blocked during study period, one patient started breathing spontaneously, and one patient develop sudden hypotension, so his vasopressor support was increased during the study period. Hence, only 50 patients met our criteria. Out of these 50 patients, 32 patients were responders and 18 were nonresponders.

Table 1 shows the comparison of demographic parameters, sedation score (Richmond Agitation Sedation), and need for vasopressor support between responders and nonresponders. All these parameters are comparable among the groups. This showed that there was no confounding effect of baseline characteristics.

Table 2 shows the comparison of hemodynamic variables between responders and nonresponders at baseline and 30 minutes and 60 minutes after administering fluid bolus. There was significant difference in $\mathrm{Cl}$ and MAP at 30 and 60 minutes after bolus fluid challenge. Also the SVV and PPV were significantly lower in responders compared to nonresponders at 30 minutes after fluid bolus.

Table 3 shows the percentage increase in $\mathrm{Cl}$ from baseline to 30 minutes and baseline to 60 minutes. It was significantly higher at both the time intervals in responders compared to nonresponders.

Table 4 shows the predictive values of different hemodynamic variables like CVP, SVV, PPV, and SPV. Among all these, SVV with a cutoff value of $14.5 \%$ has the highest sensitivity to predict fluid responsiveness following a fluid bolus in critically ill patients.

Figure 1 shows the sensitivity and specificity of SVV for responders and nonresponders. The predictive values of SVV for responders. SVV $\geq 14.5$ predicted responders correctly in $48 \%$ patients with sensitivity and specificity of $75 \%(95 \% \mathrm{Cl}=60.0-90.0)$ and $44.4 \%(95 \% \mathrm{Cl}=21.5-67.4)$, respectively.

Figure 2 shows the sensitivity and specificity of PPV for responders and nonresponders. The predictive values of PPV for responders. PPV $\leq 21.5$ predicted responders correctly in $38 \%$ patients with sensitivity and specificity of $59.4 \%(95 \% \mathrm{Cl}=42.4-76.4)$ and $50 \%(95 \% \mathrm{Cl}=26.9-73.1)$, respectively.

Figure 3 shows the sensitivity and specificity of SPV for responders and nonresponders. The predictive values of SPV for responders. SPV $\leq 14.5$ predicted responders correctly in $38 \%$ patients with sensitivity and specificity of $59.4 \%(95 \% \mathrm{Cl}=42.4-76.4)$ and $72.2 \%(95 \% \mathrm{Cl}=51.5-92.9)$, respectively.

Table 1: Comparison of demographic parameters, sedation score, and need for vasopressor support between responders and nonresponders

\begin{tabular}{llll}
\hline Parameters & Responders & Nonresponders & $p$ value \\
\hline Age (years) (mean \pm SD) & $41.88 \pm 17.74$ & $50.17 \pm 8.59$ & $0.06^{*}$ \\
Gender (percentage of males) & 59.4 & 72.2 & $0.36^{* *}$ \\
Weight (kg) (mean \pm SD) & $57.34 \pm 9.65$ & $60.72 \pm 6.7$ & 0.19 \\
Richmond Agitation Sedation Score (mean \pm SD) & $-3.91 \pm 1.17$ & $-3.67 \pm 1.18$ & 0.49 \\
Percentage of patients needing vasopressor support & 28.1 & 44.1 & $0.24^{* *}$ \\
\hline
\end{tabular}

Unpaired $t$ test, ${ }^{* *}$ Chi-square test 
Table 2: Comparison of hemodynamic variables between responder and nonresponders at baseline, and after 30 and 60 minutes of administering fluid bolus

\begin{tabular}{|c|c|c|c|c|}
\hline Parameters & Time from bolus & Responder $($ mean $\pm S D)$ & Nonresponder (mean $\pm S D)$ & $p$ value* \\
\hline \multirow[t]{3}{*}{$\mathrm{Cl}\left(\mathrm{L} /\right.$ minute $\left./ \mathrm{m}^{2}\right)$} & Baseline & $3.58 \pm 0.69$ & $3.41 \pm 0.64$ & 0.39 \\
\hline & 30 minutes & $4.24 \pm 0.73$ & $3.59 \pm 0.69$ & $0.004^{*}$ \\
\hline & 60 minutes & $4.62 \pm 0.67$ & $3.80 \pm 0.69$ & $0.0001^{*}$ \\
\hline \multirow[t]{3}{*}{ MAP $(\mathrm{mm} \mathrm{Hg})$} & Baseline & $63.56 \pm 3.10$ & $62.89 \pm 2.99$ & 0.45 \\
\hline & 30 minutes & $69.53 \pm 2.19$ & $66.06 \pm 3.15$ & $0.001^{*}$ \\
\hline & 60 minutes & $68.91 \pm 5.38$ & $64.72 \pm 5.77$ & $0.01 *$ \\
\hline \multirow[t]{3}{*}{ HR (in minutes) } & Baseline & $121.91 \pm 13.32$ & $121.67 \pm 12$ & 0.95 \\
\hline & 30 minutes & $105.69 \pm 13.4$ & $113.06 \pm 11.68$ & 0.06 \\
\hline & 60 minutes & $111.66 \pm 10.76$ & $115.83 \pm 10.77$ & 0.18 \\
\hline \multirow[t]{3}{*}{$\operatorname{CVP}\left(\mathrm{cm}\right.$ of $\left.\mathrm{H}_{2} \mathrm{O}\right)$} & Baseline & $6.97 \pm 1.93$ & $7.61 \pm 1.29$ & 0.21 \\
\hline & 30 minutes & $10.16 \pm 1.76$ & $9.28 \pm 1.6$ & 0.08 \\
\hline & 60 minutes & $9.03 \pm 2.13$ & $8.28 \pm 1.49$ & 0.19 \\
\hline \multirow[t]{3}{*}{ SVV (\%) } & Baseline & $16.09 \pm 2.18$ & $15.89 \pm 7.47$ & 0.88 \\
\hline & 30 minutes & $10.28 \pm 1.76$ & $12.28 \pm 4.42$ & $0.02 *$ \\
\hline & 60 minutes & $11.47 \pm 2.30$ & $12.00 \pm 5.40$ & 0.62 \\
\hline \multirow[t]{3}{*}{ PPV (\%) } & Baseline & $20.28 \pm 7.37$ & $23.09 \pm 8.12$ & 0.21 \\
\hline & 30 minutes & $15.28 \pm 6.94$ & $20.03 \pm 4.35$ & $0.01 *$ \\
\hline & 60 minutes & $16.99 \pm 7.16$ & $20.46 \pm 7.26$ & 0.10 \\
\hline \multirow[t]{3}{*}{ SPV (\%) } & Baseline & $13.66 \pm 4.08$ & $15.40 \pm 3.05$ & 0.12 \\
\hline & 30 minutes & $11.12 \pm 3.83$ & $12.21 \pm 3.87$ & 0.34 \\
\hline & 60 minutes & $10.66 \pm 4.01$ & $12.41 \pm 3.20$ & 0.12 \\
\hline
\end{tabular}

*Significant

Table 3: Comparison of percent increase (\%) in $\mathrm{Cl}$ from baseline to 30 and 60 minutes between responders and nonresponders

\begin{tabular}{llcl}
\hline Time periods & Responders & Nonresponders & p value \\
\hline Baseline to 30 minutes & $15.86 \pm 4.82$ & $5.29 \pm 1.74$ & $0.0001^{*}$ \\
Baseline to 60 minutes & $23.08 \pm 6.01$ & $10.51 \pm 3.82$ & $0.0001^{*}$ \\
\hline
\end{tabular}

${ }^{1}$ Unpaired $t$-test, ${ }^{*}$ significant

Table 4: Predictive values of different parameters

\begin{tabular}{llllll}
\hline & & \multicolumn{3}{c}{ Predictive values \% (95\% CI) } \\
\cline { 3 - 6 } Parameter & Cutoff value & Sensitivity & Specificity & PPV & NPV \\
\hline CVP & $7.5 \mathrm{~mm} \mathrm{Hg}$ & 62.5 & 50.0 & 69.0 & 42.9 \\
SVV & $14.5 \%$ & 75 & 44.4 & 70.6 & 50.0 \\
PPV & $21.5 \%$ & 59.4 & 50.0 & 67.9 & 40.9 \\
SPV & $14.5 \%$ & 59.4 & 72.2 & 79.2 & 50.0 \\
\hline
\end{tabular}

\section{Discussion}

This study, to our knowledge, is first ever Indian study to undertake a head-to-head comparison of three arterial waveform-derived variables in their ability to predict responsiveness to fluid bolus in critically ill hemodynamically unstable patients. It is different from previous studies in that instead of administering an arbitrary volume of fluid, irrespective of weight, as boluses, ranging from $500 \mathrm{~mL}$ to $1000 \mathrm{~mL}, 13,16-18$ we calculated the volume of fluid given as challenge on the basis of body weight $(10 \mathrm{~mL} / \mathrm{kg})$. Also, we collected data at 60 minutes, unlike previous studies, and so our study is better poised to evaluate the duration of effects of fluid challenge.

We found in our study that there was no significant difference in $\mathrm{Cl}$ between responders and nonresponders at baseline. However,
$\mathrm{Cl}$ was found to be significantly higher among responders than nonresponders at 30 minutes and 60 minutes (Table 2). Several studies had shown that rise in $\mathrm{Cl}$ was the only predictor of fluid responsiveness. 13,15

We found in our study that there was no significant difference in CVP between responders and nonresponders at all the time periods (Table 2). In their systematic review that compared CVP with measured circulating volume, the authors demonstrated a very poor relationship between CVP and blood volume as well as the inability of CVP/ $\triangle C V P$ to predict the hemodynamic response to a fluid challenge. ${ }^{15}$

In our study, we found that SVV was significantly lower among responders than nonresponders at 30 minutes. There was no significant difference in SVV between responders and 


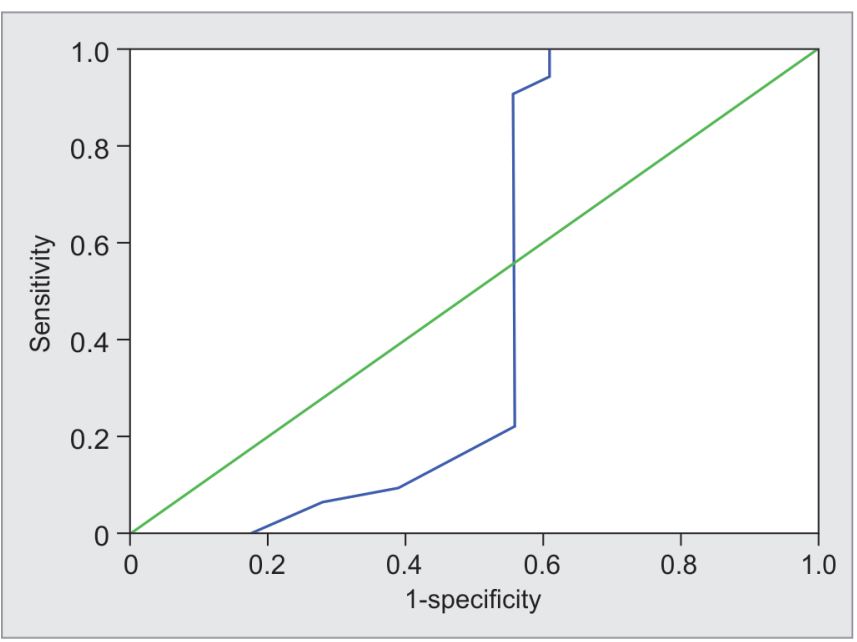

Fig. 1: Receiver operating characteristic curve showing sensitivity and specificity of stroke volume variation for responders

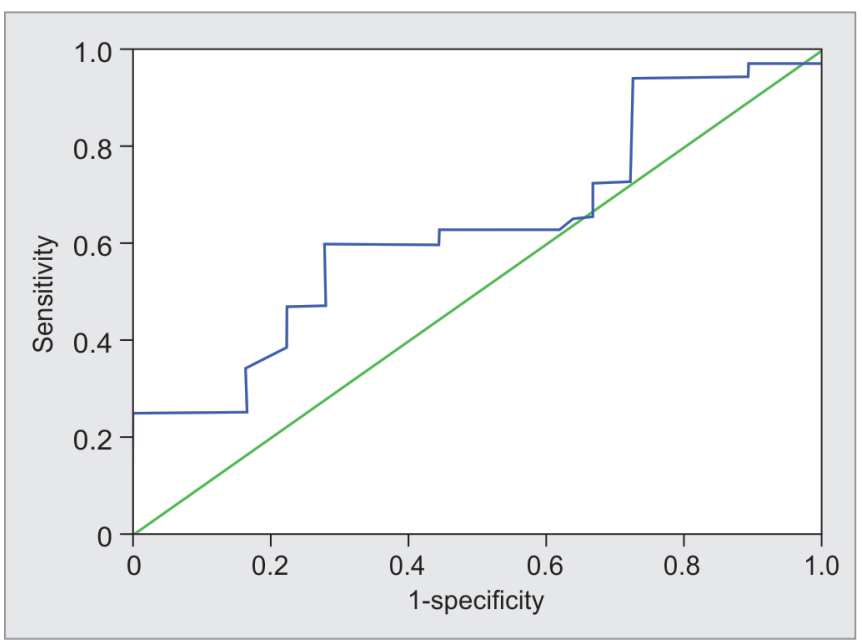

Fig. 3: Receiver operating characteristic curve showing sensitivity and specificity of systolic pressure variation for responders

nonresponders at baseline and 60 minutes (Table 2). The mean threshold value was $11.6 \pm 1.9 \%$ for the SVV in responders, which was similar to our findings, ${ }^{15}$ whereas it showed that baseline SVV $>8.15 \%$ predicted fluid responsiveness in mechanically ventilated patients with acute circulatory failure..$^{13}$ In contrast, older studies failed to appreciate the ability of SVV to predict fluid responsiveness. ${ }^{19,20}$ This could probably be explained by the fact that they used the first software version (1.01) of Vigileo-FloTrac, which has a recalibration interval of 10 minutes. This time period is too long to detect accurately the respiratory variations in the arterial pressure curve.

On comparison of PPV between the groups, we found that there was no significant difference in PPV between responders and nonresponders at baseline and 60 minutes. However, PPV was significantly lower among responders than nonresponders at 30 minutes (Table 2). Previous meta-analyzes had also shown that PPV had a high degree of diagnostic accuracy in detecting fluid responsiveness in hemodynamically unstable critically ill patients under controlled mechanical ventilation. ${ }^{21,22}$ In a systematic review of the literature of total of 649 spontaneously breathing patients assessed for fluid responsiveness, it was reported that PPV during

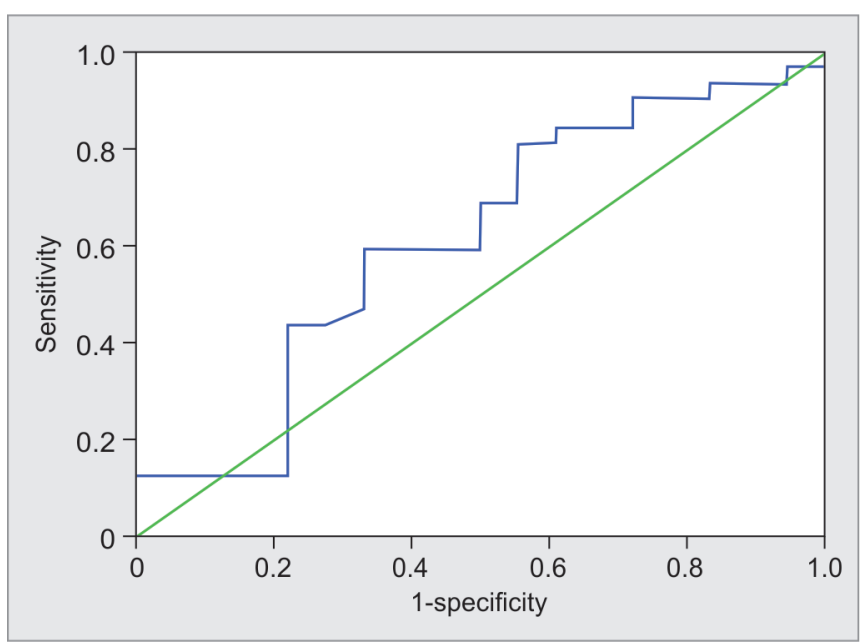

Fig. 2: Receiver operating characteristic curve showing sensitivity and specificity of pulse pressure variation for responders

the Valsalva maneuver ( $\triangle \mathrm{PPV}$ ) of $52 \%$ and passive leg raisinginduced change in stroke volume $(\triangle \mathrm{SV}$-PLR) $>13 \%$ showed the highest accuracy to predict fluid responsiveness in spontaneously breathing patients. ${ }^{23}$ Thus, PPV may be used to assess fluid responsiveness in spontaneously breathing patients too.

In our study, we found that there was no significant difference in SPV between responders and nonresponders at all the time periods. Previous studies also failed to demonstrate any relation of SPV to intravascular volume status. ${ }^{24,25}$ Further, it was reported that SPV cannot be explained by only left ventricular volume changes and other factors such as intrathoracic and airway pressure changes affect SPV. ${ }^{25}$

Table 4 shows the predictive values of SVV for responders. $S V V \geq 14.5 \%$ predicted responders correctly in $48 \%$ patients with sensitivity and specificity of 75 and $44.4 \%$, respectively. A previous prospective, interventional observer- blinded study also reported poor value of SVV in predicting fluid responsiveness with figures similar to that of our study. ${ }^{26}$ They found that $S V V \geq 8.5 \%$ predicted fluid responsiveness with sensitivity of $77 \%$, specificity of $43 \%$, positive predictive value of $84 \%$, and negative predictive value of $33 \%$. Another study reported that a threshold SVV value of $10 \%$ allowed discrimination of responders to volume expansion with a sensitivity of $82 \%$ and a specificity of $88 \%$ and they concluded that SVV predicts fluid responsiveness with an acceptable sensitivity and specificity. ${ }^{11}$

We have shown in our study that PPV $\leq 21.5 \%$ predicted responders correctly in $38 \%$ patients with sensitivity and specificity of 59.4 and $50 \%$, respectively (Table 3). Our study suggested that SPV $\leq 14.5 \%$ predicted responders correctly in $38 \%$ patients with sensitivity and specificity of 59.4 and $72.2 \%$, respectively (Table 3).

We found that SVV and PPV are both significant at 30 minutes of fluid challenge and nonsignificant at 60 minutes (Table 2). This could be probably due to the $T_{1 / 2}$ for crystalloids, which is usually 20-40 minutes. ${ }^{27}$

Our study suffers from certain important limitations like small sample size, use of crystalloids for fluid challenge, and use of hemogeneous group of patients.

Assessment of SVV requires special monitors such as Vigileo monitors with FloTrac transducers, which is expensive and may not be widely available. The Vigileo-FloTrac system, which is based on analysis of arterial pulse contour, does not need external calibration, 
dye dilution, or thermodilution. The system provides nearly beatto-beat estimate of SV and SVV. The PPV is a derivative of the arterial pulse waveform integrated in monitors of most anesthesia workstations and so more widely available with less cost. ${ }^{28}$

So, we suggest that in view of limitations of our study we need to have studies with bigger sample size and with the use of different type of fluid for challenge on heterogeneous group of patients (e.g., intraoperative, postoperative, septic shock, and pediatric).

So, to conclude, the initial assessment for fluid responsiveness in critically ill mechanically ventilated hemodynamically unstable patients should be based on SVV and PPV to prevent the complications of fluid overload and their consequences in critically ill patients.

\section{References}

1. Pinsky MR. Hemodynamic evaluation and monitoring in the ICU. Chest 2007;132(6):2020-2029. DOI: 10.1378/chest.07-0073.

2. Bredle DL, Reinhart K. Critical oxygen delivery in patients with sepsis. JAMA 1994;271(15):1158-1159. DOI: 10.1001/jama.271.15.1158.

3. Michard F, Teboul JL. Predicting fluid responsiveness in icu patients: a critical analysis of the evidence. Chest 2002;121(6):2000-2008. DOI: 10.1378/chest.121.6.2000.

4. Rosenberg AL, Dechert RE, Park PK, Bartlett RH, Nhlbi Ards NIH. Network review of a large clinical series: association of cumulative fluid balance on outcome in acute lung injury: a retrospective review of the ARDS net tidal volume study cohort. J Intensive Care Med 2009;24(1):35-46. DOI: 10.1177/0885066608329850.

5. Brandt S, Regueira T, Bracht H, Porta F, Djafarzadeh S, Takala J, et al. Effect of fluid resuscitation on mortality and organ function in experimental sepsis models. Crit Care 2009;13(6):R186. DOI: 10.1186/ cc8179.

6. Payen D, de Pont AC, Sakr Y, Spies C, Reinhart K, Vincent JL. A positive fluid balance is associated with a worse outcome in patients with acute renal failure. Crit Care 2008;12(3):R74. DOI: 10.1186/ cc6916.

7. Dubois MJ, Orellana-Jimenez C, Melot C, De Baccker D, Leeman $M$, Berre J. Albumin administration improves organ function in critically ill hypoalbuminemic patients: a prospective, randomized, controlled, pilot study. Crit Care Medicine 2006;34(10):2536-2540. DOI: 10.1097/01.CCM.0000239119.57544.0C

8. Rivers E, Nguyen B, Havstad S, Ressler J, Muzzin A, Knoblich B, et al. Early goal-directed therapy in the treatment of severe sepsis and septic shock. N Engl J Med 2001;345(19):1368-1377. DOI: 10.1056/ NEJMoa010307.

9. Levy MM, Macias WL, Russell JA, Trzaskoma BL, Vincent JL. Failure to improve during first day of therapy is predictive of 28day mortality in severe sepsis. Chest 2003;124(4):1205S. DOI: https://doi.org/10.1378/ chest.124.4_MeetingAbstracts.120S.

10. National Heart, Lung, and Blood Institute Acute Respiratory Distress Syndrome (ARDS) Clinical Trials N, Wiedemann HP, Wheeler AP, Bernard GR, Thompson BT, Hayden D, et al. Comparison of two fluid-management strategies in acute lung injury. $\mathrm{N}$ Engl J Med 2006;354(24):2564-2575. DOI: 10.1056/NEJMoa062200.

11. Cannesson M, Musard H, Desebbe O, Boucau C, Simon R, Henaine R, et al. The ability of stroke volume variations obtained with Vigileo/ FloTrac system to monitor fluid responsiveness in mechanically ventilated patients. Anesth Analg 2009;108(2):513-517. DOI: 10.1213/ ane.0b013e318192a36b.

12. Marik PE, Monnet $X$, Teboul JL. Hemodynamic parameters to guide fluid therapy. Ann Intensive Care 2011;1(1):1. DOI: 10.1186/21105820-1-1.
13. Soliman RA, Samir S, El Naggar AE, Dehley KE. Stroke volume variation compared with pulse pressure variation and cardiac index changes for prediction of fluid responsiveness in mechanically ventilated patients. EJCCM 2015;3(1):9-16. DOI: 10.1016/j.ejccm.2015.02.002.

14. Charan J, Biswas T. How to calculate sample size for different study designs in medical research? Indian J Psychol Med 2013;35(2):121-126. DOI: 10.4103/0253-7176.116232.

15. Perner A, Faber T. Stroke volume variation does not predict fluid responsiveness in patients with septic shock on pressure support ventilation. Acta Anaesthesiol Scand 2006;50(9):1068-1073. DOI: 10.1111/j.1399-6576.2006.01120.x.

16. Mayer J, Boldt J, Mengistu AM, Röhm KD, Suttner S. Goal-directed intraoperative therapy based on auto calibrated arterial pressure waveform analysis reduces hospital stay in high-risk surgical patients: a randomized, controlled trial. Crit Care 2010;14(1):R18. DOI: 10.1186/ cc8875.

17. Biais M, Bernard O, Ha JC, Degryse C, Sztark F. Abilities of pulse pressure variations and stroke volume variations to predict fluid responsiveness in prone position during scoliosis surgery. $\mathrm{Br} J$ Anaesth 2010;104(4):407-413. DOI: 10.1093/bja/aeq031.

18. De Backer D, Heenen S, Piagnerelli M, Koch M, Vincent JL. Pulse pressure variations to predict fluid responsiveness: influence of tidal volume. Intensive Care Med 2005;31(4):517-523. DOI: 10.1007/ s00134-005-2586-4.

19. Marik PE, Cavallazzi R, Vasu T, Hirani A. Dynamic changes in arterial wave form derived variables and fluid responsiveness in mechanically ventilated patients: a systematic review of the literature. Crit Care Med 2009;37(9):2642-2647. DOI: 10.1097/CCM.0b013e3181a590da.

20. de Waal EE, Rex S, Kruitwagen CL, Kalkman CJ, Buhre WF. Stroke volume variation obtained with FloTrac/Vigileo fails to predict fluid responsiveness in coronary artery bypass graft patients. Br J Anaesth 2008;100(5):725-726. DOI: 10.1093/bja/aen061.

21. Hong JQ, He HF, Chen ZY, Du ZS, Liu WF, Weng PQ, et al. Comparison of stroke volume variation with pulse pressure variation as a diagnostic indicator of fluid responsiveness in mechanically ventilated critically ill patients. Saudi Med J 2014;35(3):261-268.

22. Yang $X, D u$ B. Does pulse pressure variation predict fluid responsiveness in critically ill patients? A systematic review and meta-analysis. Crit Care 2014;18(6):650. DOI: 10.1186/s13054-0140650-6.

23. Chaves RCF, Corrêa TD, Neto AS, Bravim BA, Cordioli RL, Moreira FT, et al. Assessment of fluid responsiveness in spontaneously breathing patients: a systematic review of literature. Ann Intensive Care 2018;8(1):21. DOI: 10.1186/s13613-018-0365-y.

24. Rooke GA, Schwid HA, Shapira Y. The effect of graded hemorrhage and intravascular volume replacement on systolic pressure variation in humans during mechanical and spontaneous ventilation. Anesth Analg 1995;80(5):925-932. DOI: 10.1097/00000539-199505000-00012.

25. Denault AY, Gasior TA, Gorscan 3rd J, Mandarino WA, Deneault LG, Pinsky MR. Determinants of aortic pressure variation during positive pressure ventilation in man. Chest 1999;116(1):176-186. DOI: 10.1378/ chest.116.1.176.

26. Lahner D, Kabon B, Marschalek C, Chiari A, Pestel G, Kaider A, et al. Evaluation of stroke volume variation obtained by arterial pulse contour analysis to predict fluid responsiveness intraoperatively. Br J Anaesth 2009;103(3):346-351. DOI: https://doi.org/10.1093/bja/ aep200.

27. Hahn RG, Lyons $G$. The half-life of infusion fluids. an educational review. Eur J Anaesthesiol 2016;33(7):475-482. DOI: 10.1097/ EJA.0000000000000436.

28. Rathore A, Singh S, Lamsal R, Taank P, Paul D. Validity of pulse pressure variation (PPV) compared with stroke volume variation (SVV) in predicting fluid responsiveness. Turk J Anaesthesiol Reanim 2017;45(4):210-217. DOI: 10.5152/TJAR.2017.04568. 